\title{
Assessing the cost-effectiveness of drug and lifestyle intervention following opportunistic screening for pre-diabetes in primary care
}

\author{
M. Y. Bertram • S. S. Lim • J. J. Barendregt • T. Vos
}

Received: 7 September 2009 /Accepted: 16 December 2009/Published online: 5 February 2010

(C) Springer-Verlag 2010

\begin{abstract}
Aims/hypothesis This study aims to evaluate the costeffectiveness of a screening programme for pre-diabetes, which was followed up by treatment with pharmaceutical interventions (acarbose, metformin, orlistat) or lifestyle interventions (diet, exercise, diet and exercise) in order to prevent or slow the onset of diabetes in those at high risk. Methods To approximate the experience of individuals with pre-diabetes in the Australian population, we used a microsimulation approach, following patient progression through diabetes, cardiovascular disease and renal failure. The model compares costs and disability-adjusted life years lived in people identified through an opportunistic screening programme for each intervention compared with a 'do nothing' scenario, which is representative of current practice. It is assumed that the effect of a lifestyle change will decay by $10 \%$ per year, while the effect of a pharmaceutical intervention remains constant throughout use.

Results The most cost-effective intervention options are diet and exercise combined, with a cost-effectiveness ratio of AUD 22,500 per disability-adjusted life year (DALY) averted, and metformin with a cost-effectiveness ratio of
\end{abstract}

Electronic supplementary material The online version of this article (doi:10.1007/s00125-010-1661-8) contains supplementary material, which is available to authorised users.

M. Y. Bertram $(\bowtie) \cdot$ J. J. Barendregt $\cdot$ T. Vos

Centre for Burden of Disease and Cost-Effectiveness,

School of Population Health, The University of Queensland,

Herston Rd, Herston,

Queensland 4006, Australia

e-mail: m.bertram@sph.uq.edu.au

\section{S. S. Lim}

Centre for Health Metrics and Evaluation,

The University of Washington,

Seattle, WA, USA
AUD 21,500 per DALY averted. The incremental addition of one intervention to the other is not cost-effective.

Conclusions/interpretation Screening for pre-diabetes followed by diet and exercise, or metformin treatment is costeffective and should be considered for incorporation into current practice. The number of dietitians and exercise physiologists needed to deliver such lifestyle change interventions will need to be increased to appropriately support the intervention.

Keywords Cost-effectiveness - Economic evaluation . Pre-diabetes $\cdot$ Screening
Abbreviations
CVD Cardiovascular disease
DALY Disability-adjusted life years

\section{Introduction}

Diabetes is a leading cause of morbidity and mortality worldwide [1]. In Australia, 9.6\% of fatal and non-fatal health loss measured in disability-adjusted life years (DALYs) and $12.6 \%$ of all deaths are attributed to type 2 diabetes [2]. Increased body weight is the main risk factor for type 2 diabetes mellitus and in 2003 accounted for 5\% of total DALYs due to diabetes [2]. Direct healthcare costs are estimated to be AUD 835 million annually, representing $1.7 \%$ of total health expenditure [3]. If the expenditure on cardiovascular disease (CVD) and renal failure that is attributable to diabetes is included, the figure would rise to $2.2 \%$ of total expenditure. Expenditure on diabetes is projected to increase fourfold between 2003 and 2033 [4]. Therefore, prevention of the health and economic burden of 
diabetes is a major challenge for public health policy makers.

Pre-diabetes is defined as having impaired fasting glucose or impaired glucose tolerance, but not (yet) having diabetes. A recent meta-analysis of all the available interventions to prevent the onset of diabetes in people with pre-diabetes shows a range of pharmaceutical and lifestyle interventions to be efficacious [5]. Despite impressively reducing diabetes incidence, these interventions are not available to people with pre-diabetes in Australia. Reducing the onset of diabetes could have beneficial impacts on overall population health and on the economic burden of healthcare. A number of studies have evaluated the cost-effectiveness of diabetes prevention, with two recent ones evaluating a range of screening and treatment programmes [6, 7]. The study by Gillies et al. [7] evaluated lifestyle interventions and combined pharmacotherapies in the UK population. The Colagiuri study was undertaken in the Australian population, but analysed a lifestyle intervention only [6]. No study has yet compared individual pharmacotherapies with different lifestyle interventions in an Australian population.

The aim of this study was to evaluate the cost-effectiveness of a screening programme for pre-diabetes followed up by treatment with pharmaceutical interventions (acarbose, metformin or orlistat) and lifestyle interventions (diet, exercise, or diet and exercise combined) in the Australian population. This enables comparison of different interventions in order to identify the most cost-effective prevention strategy available. This study forms part of a larger project, the Assessing Costeffectiveness - Prevention Project, which aims to evaluate the cost-effectiveness of 100 preventive interventions for noncommunicable disease [8].

\section{Methods}

Using an epidemiological modelling approach, we calculated the cost-effectiveness of screening to identify individuals with pre-diabetes, followed up by treatment with a pharmaceutical or lifestyle intervention. The screening programme was designed to target the Australian population, and specifically those who were older than 45 years and did not have diabetes, but did have risk factors for the disease.

Epidemiological modelling The methods are described here in brief, with detailed methods available in the Electronic supplementary material (ESM). The model developed by us is a discrete-time micro-simulation model, which estimates the health impact and costs of preventing diabetes among people with pre-diabetes. Micro-simulation models disease by simulating disease at the level of the individual. It creates individual life histories by simulating various health states over the individual's lifetime. Micro-simulation was used to allow flexibility in modelling co-morbidities without restraining individuals to pre-defined combinations of events. We used a discrete time period of 1 year for each cycle. Individuals were simulated until death or age 100 , whichever would have occurred first.

Transitions were explicitly modelled for four health states: (1) glucose tolerance; (2) CVD; (3) stroke; and (4) renal failure in diabetes. CVD, stroke and renal failure are all either present or absent. For CVD and stroke, incident cases may die within the 1st year, either due to increased mortality in the 28 days following their event, or due to the case-fatality in the remainder of the year. Within the glucose tolerance health state, an individual may have normal glucose tolerance, pre-diabetes or diabetes. An individual can have either impaired glucose tolerance or diabetes, but not both at the same time. Individuals may remit from pre-diabetes to normal glucose tolerance; however, it is assumed that once a patient is diagnosed with diabetes, he or she cannot remit from this state. In successive time periods, the individual can move between these states. Figure 1 describes the sub-states and possible transitions for each of the health states. Note that while any individual may become an incident case of CVD or stroke, only diabetic renal failure is considered in the model.

Using the demographic characteristics of the Australian population along with current epidemiology of pre-diabetes and associated diseases in Australia, we created 8,000 individual life-histories. Random number draws determined whether an individual experienced an incident event, remitted to normal glucose levels or died. A random number between 0 and 1 was drawn for each potential transition. If the number fell within the incidence, remission or mortality range (i.e. between 0 and the transition probability), the individual would transit to a new state, otherwise he/she would remain in his/her health state to date. Transitions could occur between multiple states in any given year. Thus an individual could, for example, experience stroke and renal failure in the same year. Transition probabilities are shown in the ESM Table 2.

Epidemiological data used in the model came from a number of sources. The main source was the Australian burden of disease and injury study [2]. Other sources include the Australian diabetes, obesity and lifestyle study (AusDiab) [9] and vital registration data from the Australian Bureau of Statistics [10]. Disease treatment costs came from the Australian Institute of Health and Welfare (AIHW) disease costs and impact study (DCIS) [3]. Full details on epidemiological data can be found in the ESM.

A complete set of epidemiological variables, including incidence, prevalence, duration, remission and case-fatality for pre-diabetes was not available. Using the prevalence and incidence from the AusDiab Study [9] along with our own 
Fig. 1 Transition diagram of the diabetes micro-simulation model. T1-12 present potential transitions. Definitions and values are shown in ESM Tables 1 and 2
Glucose tolerance (S1)

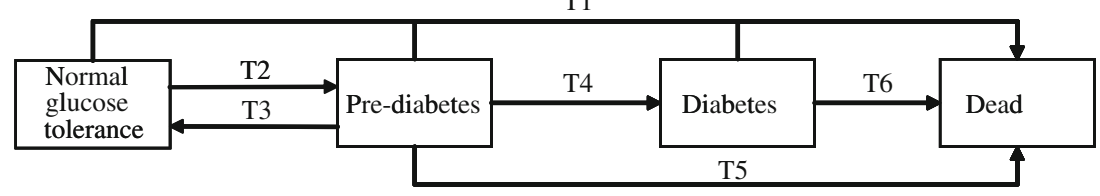

Ischaemic heart disease (S2)

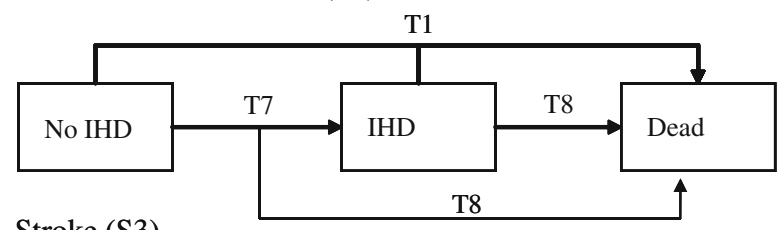

Stroke (S3)

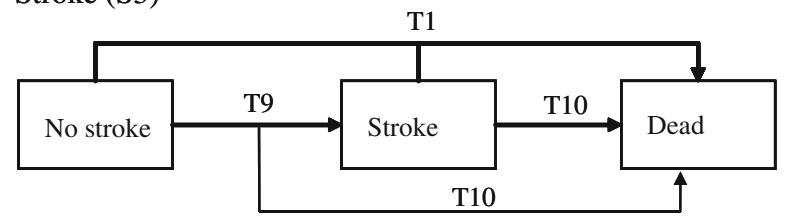

Diabetic renal disease (S4)

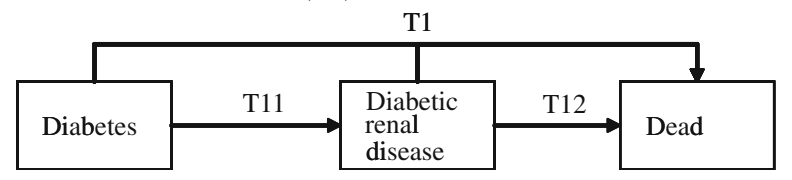

meta-analysis of the increase in all-cause mortality, we used DisMod [11] to estimate a full set of epidemiological variables (M. Y. Bertram and T. Vos, unpublished results). The DisMod outputs were also used to determine the duration of pre-diabetes to establish whether screening is viable.

The model measures DALYs in the population in the presence or absence of an intervention. We used disability weights derived from the Australian burden of disease study to adjust each year in the model to reflect the equivalent time spent in full health [2]. An individual's disability weight is dependent upon his or her current health state. Total intervention costs and the potential cost-savings due to disease avoidance (cost-offsets) are also recorded. All costs and benefits are referenced to 2003 and calculated using a $3 \%$ discount rate. Costs are measured in Australian dollars.

Screening A screening programme was modelled based upon the National Health and Medical Research Council guidelines for screening of diabetes [12]. These guidelines were applied to the population in the AusDiab study and the rate of identification of people with pre-diabetes was calculated [13]. Inclusion criteria of the opportunistic screening programme included: (1) age $>55$ years; or (2) age $>45$ plus high BMI, family history of type 2 diabetes or hypertension; or (3) people from 'high-risk' groups (e.g. Indigenous Australians and women who suffered from gestational diabetes). In the model, these people would be eligible for opportunistic screening for pre-diabetes during a visit to a GP for an unrelated condition. The number of people seeing a GP every year was based on the report
General Practice in Australia 2004 [14]. At the initial GP visit, patients are invited to participate in screening, which involves a plasma glucose test at this visit. If this test indicates pre-diabetes may be present, the patient is requested to attend for an OGTT for diagnosis.

Interventions We analysed the impact of six alternative interventions: three pharmaceutical therapies (acarbose, metformin and orlistat) and three lifestyle interventions (diet alone, exercise alone, and diet and exercise). Intervention efficacy was based on a systematic review and meta-analysis [5]. This study provided relative risks (RRs) of a reduction in diabetes incidence for a range of interventions including diet, exercise, diet and exercise, and all pharmaceutical interventions combined. Using the trials identified, we replicated the meta-analyses of individual pharmaceuticals available in Australia. The lifestyle interventions involved an intensive programme of visits to a dietitian, exercise physiologist or both, visits being once a week for 1 month, monthly for 3 months and at threemonthly intervals thereafter.

The RRs of reduction in diabetes incidence of those with pre-diabetes (Table 1) were calculated on an intention-totreat basis. Using this value, along with the published follow-up rate for each intervention, we were able to calculate the RR in those who participate [5, 15-22]. This enabled us to account for the lower adherence expected under routine health service conditions compared with clinical trials. This RR was applied to those treated in the model, following their identification through screening. The 
Table 1 Intervention efficacy and yearly costs of delivering each intervention
Costs are measured per patient identified and treated

\begin{tabular}{lccc}
\hline Intervention & RR (SE) from meta-analysis & Government cost (AUD) & Patient cost (AUD) \\
\hline Diet and exercise & $0.486(0.079)$ & 126 & 265 \\
Exercise & $0.488(0.213)$ & 121 & 164 \\
Diet & $0.667(0.161)$ & 102 & 118 \\
Acarbose & $0.602(0.273)$ & 248 & 291 \\
Metformin & $0.679(0.232)$ & 58 & 200 \\
Orlistat & $0.437(0.232)$ & 1,290 & 320 \\
\hline
\end{tabular}

efficacy of diet and exercise interventions was assumed to decay over time at a rate of $10 \%$ per year [23], while the pharmaceutical interventions were anticipated to have the same efficacy throughout use as long as people remained adherent. A decay of $10 \%$ per year causes the intervention to lose its effect after 10 years.

Costs for each intervention (Table 1) were calculated on a yearly basis, including costs of GP visits, medication, monitoring costs and visits to other health professionals including dietitians and exercise physiologists. Values for each of these components come from the Australian Medical Benefits Schedule and Pharmaceutical Benefits Schedule $[24,25]$. The costing perspective is the healthcare system, which in Australia is largely funded by the government as a third-party payer. Patient contributions to medication prescribed during GP visits are calculated using data provided by the Commonwealth government (unpublished data, Commonwealth Government, Canberra, Australia). Time and travel costs attributed to the patient are also calculated. Travel costs are based upon the average distance travelled to see a GP, weighted according to the Australian population living in urban, rural and remote areas $[26,27]$. Time costs incorporate time spent travelling to a GP, as well as waiting time, and are calculated using the population average hourly wage.

The impact of uncertainty around key input values (Table 2) on the main outcome measures was estimated by calculating 1,000 second-order simulations using @ RISK (Palisade Corporation, Ithaca, NY, USA). Common random numbers for the 8,000 individuals determined transitions for each of the 1,000 uncertainty iterations. As a result, the reported $95 \%$ uncertainty ranges reflect variability due to uncertainty in the input values only and not due to first-order (or stochastic) variation in disease progression in the population.

\section{Results}

Approximately six million Australians are currently eligible for screening. Of these, we anticipate that in the first year of a screening programme two million would be invited to participate, with 580,000 people being identified with pre- diabetes. This would result in a total cost to the government of AUD 94 million and direct costs to the patient of AUD 17 million. A further AUD 82 million in time and travel costs can be attributed to the patients. Total yearly costs for each intervention following screening include those attributed to government and out-of-pocket costs to the patient (Table 3). The number of DALYs averted and cases of diabetes prevented are shown in Table 3.

The most cost-effective interventions are diet and exercise, and metformin (Table 3). The chance of an intervention falling below AUD 50,000/DALY is included, as this represents the willingness-to-pay threshold commonly applied in Australia [28]. Diet and exercise and metformin have similar cost-effectiveness ratios, but different uncertainty, as shown in an acceptability curve (Fig. 2) indicating the probability that the cost-effectiveness ratio falls below a given threshold of cost/DALY. This demonstrates the impact that the uncertainty has on each of the interventions. In an incremental analysis, the addition of metformin and diet and exercise was not cost-effective, with a median cost-effectiveness ratio of AUD 67,000/ DALY when adding diet and exercise to metformin or AUD 81,000/DALY when adding metformin to diet and exercise.

Sensitivity analysis was undertaken around the effect of adding a second OGTT to the opportunistic screening programme, using information from Mooy et al. [29] that up to $49 \%$ of incident pre-diabetes cases would be reclassified if a second test was undertaken. As the additional OGTT only increases the cost of identifying a case, but does not affect the ratio between treatment cost and benefits, the overall effect on the cost-effectiveness ratio was to increase the median cost-effectiveness ratio for both metformin and diet and exercise to AUD 29,000/ DALY, which does not significantly alter the conclusions of the study. A regression analysis of the variables with the largest impact on health outcomes and intervention costs indicated that the RR of intervention efficacy was the most influential input variable, followed by the likelihood of participation and assumptions surrounding GP participation. The RR of interventions was taken from international literature and was assumed not to differ across populations. However, participation rates of GPs are likely to differ between populations. 
Table 2 Uncertainty surrounding key input variables in the model

IHD, ischaemic heart disease

\begin{tabular}{llll}
\hline Variables & Distribution & Mean \pm SE & Source \\
\hline $\begin{array}{lll}\text { RR stroke in CHD } \\
\text { Men }\end{array}$ & Log normal & $1.32 \pm 0.265$ & Busselton Study [32] \\
Women & Log normal & $1.88 \pm 0.564$ & \\
RR CHD in stroke & & & Busselton Study [32] \\
Men & Log normal & $2.64 \pm 0.171$ & \\
Women & Log normal & $2.85 \pm 0.099$ & Framingham Study [33] \\
RR IHD in diabetes & & & \\
Men & Log normal & $1.41 \pm 0.157$ & Framingham Study [33] \\
$\quad$ Women & Log normal & $1.75 \pm 0.240$ & \\
RR stroke in diabetes & & & \\
$\quad \begin{array}{l}\text { Men } \\
\text { Women }\end{array}$ & Log normal & $1.34 \pm 0.270$ & NEMESIS [34, 35] \\
28-day case-fatality rate, & Log normal & $1.72 \pm 0.385$ & NEMESIS [34, 35] \\
ischaemic stroke & Normal & $0.12 \pm 0.023$ & \\
28-day case-fatality rate, & Normal & $0.46 \pm 0.077$ & \\
\hline
\end{tabular}

\section{Discussion}

Several cost-effective interventions are available for prediabetes, with either pharmacological or lifestyle interventions possible. Screening followed by metformin or by diet and exercise falls below the AUD 50,000/DALY willingness-to-pay threshold, making either intervention cost-effective in the Australian context.

We used a modelling approach to combine information from various sources, as an alternative to running a large trial with a long follow-up. Assumptions made include that people must pass through the pre-diabetes state in order to be an incident case of diabetes. Recent analysis of a British cohort supports this assumption, with the investigators finding a linear trend in fasting glucose levels over time, followed by a 'steep quadratic increase' in the 3 years prior to diagnosis of diabetes, indicating that all people pass through pre-diabetes [30]. Use of the Framingham equations [33] to estimate relative risks of CVD events is a weakness of the modelling approach. It would be more accurate to use follow-up studies of people in Australia at each glucose level and monitor their cardiovascular outcomes. The only source of data that may enable this is AusDiab [9], which asked for a history of CVD events by self-report at two time points and collected information on cause of death. However, there are inherent problems with using self-reported data, such as recall bias; also the numbers of events are small when stratified by age, sex and glucose status. Modelled data using Framingham equations are the best alternative.

Aside from the model itself, there are some limitations in the analysis. First, screening targeted at pre-diabetes will also identify some undiagnosed cases of diabetes. The AusDiab Study in Australia found that up to half of all people with diabetes were undiagnosed, whereas in the USA the National Health and Nutrition Examination Survey in $2005 / 06$ reported that $40 \%$ of diabetes was

Table 3 Effects of six interventions as indicated per 100,000 identified cases of pre-diabetes

\begin{tabular}{lccccc}
\hline Intervention & DALYs averted & $\begin{array}{l}\text { Diabetes cases } \\
\text { avoided }\end{array}$ & $\begin{array}{l}\text { CER } \\
\text { (AUD/DALY) }\end{array}$ & $\begin{array}{l}\text { 95\% uncertainty } \\
\text { interval }\end{array}$ & $\begin{array}{l}\text { CER<AUD50,000/DALY } \\
\text { averted }^{\mathrm{b}}\end{array}$ \\
\hline Diet plus exercise & 4,730 & 8,150 & 23,000 & $19,000-35,000$ & 100 \\
Exercise & 4,000 & 6,650 & 30,000 & $23,000-89,000$ & 86 \\
Diet & 2,290 & 4,070 & 38,000 & $23,000-148,000$ & 75 \\
Acarbose & 5,700 & 13,140 & 37,000 & $25,000-134,000$ & 76 \\
Metformin & 4,290 & 9,900 & 22,000 & $17,000-36,000$ & 100 \\
Orlistat & 6,880 & 15,830 & 100,000 & $94,000-130,000$ & 0 \\
Metformin+diet plus exercise & 1,100 & 2,490 & 81,000 & $14,000-130,000$ & 64 \\
\hline
\end{tabular}

${ }^{\text {a }}$ Median cost-effectiveness ratio (CER)

${ }^{\mathrm{b}}$ Probability (\%)

${ }^{\mathrm{c}}$ Incremental addition of metformin 
Fig. 2 Acceptability curves displaying various willingness-topay values for each DALY averted and the probability of an intervention being considered cost-effective at each willingness to pay threshold

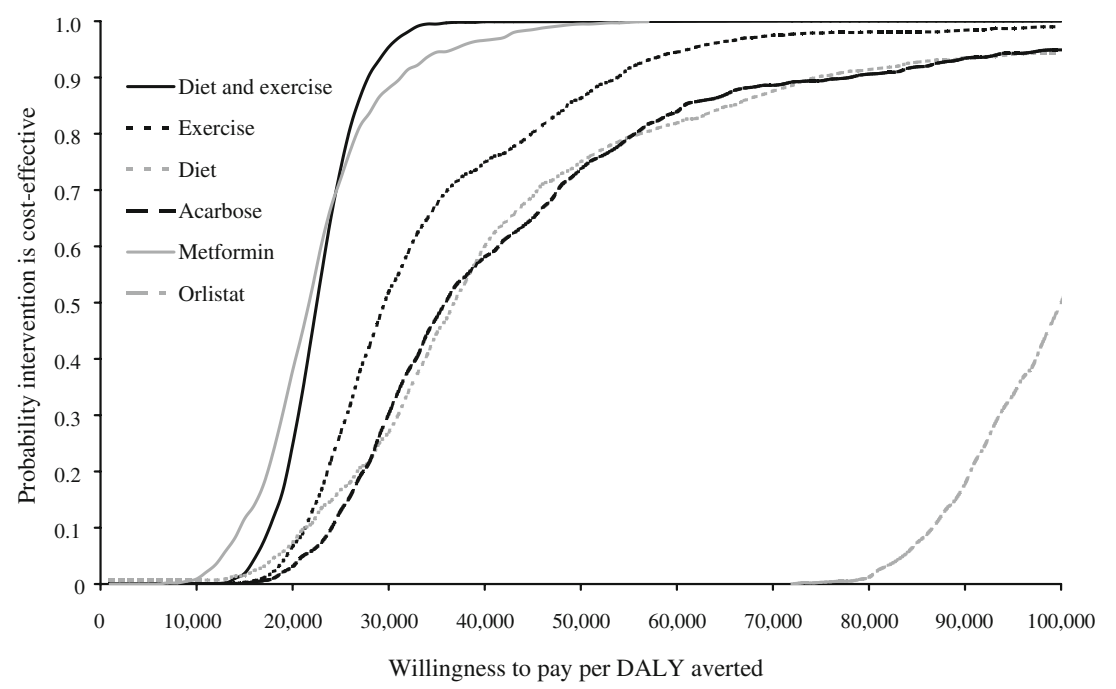

undiagnosed [13, 31]. Increased case finding would increase diabetes treatment costs, but would also lead to health benefits. Second, lifestyle interventions may have broader health consequences beyond those related to glucose status; these broader consequences have not been quantified. An estimated $7.5 \%$ of the disease burden is attributable to increased BMI [2]. The model explicitly accounts for diabetes and CVD, which are responsible for $84 \%$ of the burden due to increased BMI. Given that a diet and exercise intervention could also have an effect on BMI, marginally higher health gains from other diseases not included in this analysis are possible. There may also be an independent effect on CVD, mediated by a reduction in blood pressure and cholesterol, which would lead to greater health gain and thus more favourable cost-effectiveness ratios.

Although we accounted for adherence to medication, the values used are from randomised controlled trials, which generally recruit motivated participants. As this analysis is based on opportunistic screening of the general population, participation rates are likely to be lower than those from the trials. This would reduce the overall health gain due to intervention, but would also reduce the costs of providing the intervention. A small increase in the cost per identified and treated patient would be expected. However, as this is a small component of the total costs, the cost-effectiveness ratios would be largely unaffected.

Technical cost-effectiveness analysis should be combined with other approaches that facilitate 'due process' [8]. Issues relating to the feasibility, sustainability and acceptability associated with the implementation of these interventions were considered alongside the results of the technical analysis in the Assessing Cost-effectiveness: Prevention Project [8]. The pertinent issues include workforce capacity and feasibility of GP participation.

The current workforce capacity of dietitians and exercise physiologists is unlikely to be able to meet the increased demand for their services ensuing from a screening programme. Therefore more training places would be required, which in turn would depend on the teaching capacity in universities. Given that increased efficacy and efficiency were found for the combination of diet and exercise, a subsidy could be based on attendance at dietitian and exercise physiologist practices, with joint practices of dietitians and exercise physiologists possibly needed to ensure that patients participate appropriately.

The biggest feasibility issue would be levels of GP participation in the screening programme. Currently, the modelled participation rates are optimistic. However, changing these rates would not change the cost-effectiveness ratio, only the total costs and total benefits, by reducing the number of people identified and participating.

Lifestyle modification in people with pre-diabetes is cost-effective and should be recommended if workforce capacity can be increased in order to appropriately deliver the intervention. A government subsidy for exercise physiologists and dietitians would encourage participation. The likely financial cost to the government would be up to AUD300 million annually (based on current cost to the patient), dependent upon the level of subsidy given and the number of visits supported per year. The use of metformin following identification is also cost-effective and should be considered as an alternative treatment regimen. Metformin would be less of a financial burden to the government. The interventions should not be used in combination as this is not cost-effective.

Future research addressing the increased case-finding of diabetes due to screening for pre-diabetes would answer important questions relating to the financial implications of a screening programme such as this one. Also, analysis of the independent effect of lifestyle modification interventions on CVD would enable more accurate calculation of the health gains of screening for pre-diabetes. 
Acknowledgements The Assessing Cost-effectiveness: Prevention Project is funded by an Australian National Health and Medical Research Council Health Services Research Grant (NHMRC HSR Grant 331558). M. Y. Bertram was supported by a University of Queensland PostGraduate Research Scholarship. The authors wish to acknowledge the AusDiab study authors for providing access to their data.

Duality of interest The authors declare that there is no duality of interest associated with this manuscript.

\section{References}

1. Wild S, Roglic G, Green A, Sicree R, King H (2004) Global prevalence of diabetes: estimates for the year 2000 and projections for 2030. Diabetes Care 27:1047-1053

2. Begg SJ, Vos T, Barker B, Stanley L, Lopez AD (2008) Burden of disease and injury in Australia in the new millenium: measuring health loss from diseases, injuries, and risk factors. Med J Aust $188: 36-40$

3. Australian Institute of Health and Welfare (2007) Disease Costs and Impact Study. Australian Institute of Health and Welfare, Canberra

4. Begg S, Vos T, Goss J, Mann N (2008) An alternative approach to projecting health expenditure in Australia. Aust Health Rev 32:148-155

5. Gillies CL, Abrams KR, Lambert PC et al (2007) Pharmacological and lifestyle interventions to prevent or delay type 2 diabetes in people with impaired glucose tolerance: systematic review and meta-analysis. BMJ 334:299

6. Colagiuri S, Walker A (2008) Using an economic model of diabetes to evaluate prevention and care strategies in Australia. Health Aff 27:256-268

7. Gillies CL, Lambert PC, Abrams KR et al (2008) Different strategies for screening and prevention of type 2 diabetes in adults: cost effectiveness analysis. BMJ 336:1180-1185

8. Carter R, Vos T, Moodie M, Haby M, Magnus A, Mihalopoulis C (2008) Priority setting in health: origins, description and application of the Australian Assessing Cost Effectiveness (ACE) Initiative. Expert Review of Pharmacoeconomics and Outcomes Research 8:593-617

9. Dunstan DW, Zimmet PZ, Welborn TA et al (2002) The Australian Diabetes, Obesity and Lifestyle Study (AusDiab) methods and response rates. Diabetes Res Clin Pract 57:119-129

10. No authors listed (2005) Causes of death in Australia 2003. Australian Bureau of Statistics, Canberra, Australia

11. Barendregt J, van Oortmarssen G, Vos T, Murray C (2003) A generic model for the assessment of disease epidemiology: the computational basis of DisMod II. Population Health Metrics 1:4-11

12. Colagiuri S, Zimmet P, Welborn T et al. (2005) Part 3: case detection and diagnosis. In: National evidence based guidelines for the management of type 2 diabetes mellitus, pp 6-7

13. Colagiuri S, Hussain Z, Zimmet P, Cameron A, Shaw J (2004) Screening for type 2 diabetes and impaired glucose metabolism. The Australian experience. Diabetes Care 27:367-371

14. No authors listed (2005) General practice in Australia: 2004. Department of Health and Ageing, Budget and Performance Branch, Primary Care Division, Canberra, Australia

15. Chiasson JL, Josse RG, Gomis R et al (2004) Acarbose for the prevention of type 2 diabetes, hypertension and cardiovascular disease in subjects with impaired glucose tolerance: facts and interpretations concerning the critical analysis of the STOPNIDDM Trial data. Diabetologia 47:969-975

16. Diabetes Prevention Program Research Group (2002) The Diabetes Prevention Program (DPP): description of lifestyle intervention. Diabetes Care 25:2165-2171
17. Fang YS, Li TY, Chen SY (2004) Effect of medicine and nonmedicine intervention on the outcomes of patients with impaired glucose tolerance: 5 year follow up. Zhongguo lin chuang kang fu $8: 6562-6563$

18. Heymsfield SB, Segal KR, Hauptman J et al (2000) Effects of weight loss with orlistat on glucose tolerance and progression to type 2 diabetes in obese adults. Arch Intern Med 160:1321-1326

19. Li CL, Pan CY, Lu JM et al (1999) Effect of metformin on patients with impaired glucose tolerance. Diabet Med 16:477-481

20. Pan CY, Gao Y, Chen JW et al (2003) Efficacy of acarbose in Chinese subjects with impaired glucose tolerance. Diabetes Res Clin Pract 61:183-190

21. Ramachandran A, Snehalatha C, Mary S et al (2006) The Indian Diabetes Prevention Programme shows that lifestyle modification and metformin prevent type 2 diabetes in Asian Indian subjects with impaired glucose tolerance (IDPP-1). Diabetologia 49:289297

22. Torgerson JS, Hauptman J, Boldrin MN, Sjostrom L (2004) XENical in the prevention of diabetes in obese subjects (XENDOS) study: a randomized study of orlistat as an adjunct to lifestyle changes for the prevention of type 2 diabetes in obese patients. Diabetes Care 27:155-161

23. Lindström J, Ilanne-Parikka P, Peltonen M et al (2006) Sustained reduction in the incidence of type 2 diabetes by lifestyle intervention: follow-up of the Finnish Diabetes Prevention Study. Lancet 368:1673-1679

24. No authors listed (2003) Pharmaceutical benefits schedule. Department of Health and Ageing, Canberra, Australia

25. No authors listed (2003) Medical benefits schedule. Department of Health and Ageing, Canberra, Australia

26. Bamford E, Dunne L, Taylor D, Symon B, Hugo G, Wilkinson D (1999) Accessibility to general practitioners in rural South Australia. A case study using geographic information system technology. Med J Aust 171:614-616

27. Rankin S, Hughes-Anderson W, House J et al (2002) Rural residents' utilisation of health and visiting specialist health services. Rural Remote Health 2:119

28. George B, Harris A, Mitchell A (2001) Cost-effectiveness analysis and the consistency of decision making. Evidence from pharmaceutical reimbursement in Australia (1991-1996). Pharmacoeconomics 19:1103-1109

29. Mooy J, Grootenhuis P, de Vries H et al (1996) Intra-individual variation of glucose, specific insulin and proinsulin concentrations measured by two oral glucose tolerance tests in a general Caucasian population: the Hoorn Study. Diabetologia 39:298-305

30. Tabák A, Jokela M, Akbaraly T, Brunner E, Kivimäki M, Witte D (2009) Trajectories of glycaemia, insulin sensitivity, and insulin secretion before diagnosis of type 2 diabetes: an analysis from the Whitehall II Study. Lancet 373:2215-2221

31. Cowie CC, Rust KF, Ford ES et al (2009) Full accounting of diabetes and pre-diabetes in the U.S. population in 1988-1994 and 2005-2006. Diabetes Care 32:287-294

32. Knuiman MW, Vu HT (1996) Risk factors for stroke mortality in men and women: the Busselton Study. J Cardiovasc Risk 3:447452

33. D'Agostino RB, Russell MW, Huse DM et al (2000) Primary and subsequent coronary risk appraisal: new results from the Framingham Study. Am Heart J 139:272-281

34. Dewey HM, Sturm J, Donnan GA et al (2003) Incidence and outcome of subtypes of ischaemic stroke: initial results from the North East Melbourne Stroke Incidence Study (NEMESIS). Cerebrovasc Dis 15:133-139

35. Thrift AG, Dewey HM, Macdonell RA, McNeil JJ, Donnan GA (2001) Incidence of the major stroke subtypes: initial findings from the North East Melbourne Stroke Incidence Study (NEMESIS). Stroke 32:1732-1738 